\title{
INDEX OF VOLUME 44
}

\section{AUTHORS}

Albert, A. A. A quadratic form problem in the calculus of variations, 250.

Non-cyclic algebras with pure maximal subfields, 576.

A note on normal division algebras of prime degree, 649.

The Chicago conference and seminar on algebra, 756.

Allen, E. S. See Reviews, under Woodger.

Aronszajn, N. Quelques remarques sur les relations entre les notions d'écart régulier et de distance, 653.

Ayres, W. L. Report of the April meeting in Chicago, 457.

Baer, R. The applicability of lattice theory to group theory, 817.

Basoco, M. A. See Reviews, under Nicolesco.

Bateman, H. See Reviews, under Mattioli.

Beckenbach, E. F. A relative of the lemma of Schwarz, 698.

Beeger, N. G. W. H. On some new congruences in the theory of Bernoulli's numbers, 684.

Bell, E. T. See Reviews, under Zassenhaus.

Bennett, A. A. See Reviews, under Osgood, Roth.

Birkhoff, G. Lattices and their applications, 793.

See Reviews, under Volterra.

Boas, R. P., and Tukey, J. W. A note on linear functionals, 523.

Bochner, S. See Reviews, under Thomas.

Bohnenblust, H. F., and Sobczyk, A. Extensions of functionals on complex linear spaces, 91.

Brinkmann, H. W. See Reviews, under Albert.

Bristow, L. Expansion of functions in solutions of functional equations, 874.

Buchanan, H. E. See Reviews, under Whittaker.

Church, A. The constructive second number class, 224.

- See Reviews, under Huntington.

Churchill, R. V. See Reviews, under Doetsch.

Clements, G. R. See Reviews, under British Association for the Advancement of Science.

Coble, A. B. Frank Morley -in memoriam, 167.

Copeland, A. H. See Reviews, under Mineur.

Coral, M. A generalization of a property of harmonic functions, 587.

Cramlet, C. M. Note on integrability conditions of implicit differential equations, 107. Differential invariant theory of alternating tensors, 110.

Creedy, F. See Reviews, under Russell.

Davis, H. T. See Reviews, under G. Guillaume, Tinbergen.

DeCicco, J. J. See Kasner, E.

Dilworth, R. P. Abstract residuation over lattices, 262.

Dodd, E. L. See Reviews, under Böhm.

Doob, J. L. See Reviews, under Bachelier, Lévy.

Dresch, F. W. Index numbers and the general economic equilibrium, 134.

Dresden, A. See Reviews, under Contributions to the Calculus Variations.

Dressel, F. G. A note on Fredholm-Stieltjes integral equations, 434.

Duffin, R. J. See Schaeffer, A. C.

Duffin, R. J., and Schaeffer, A. C. Some properties of functions of exponential type, 236.

Emmons, H. See Reviews, under Stumpff.

Erdös, P., and Grünwald, G. Note on an elementary problem of interpolation, 515. 
Erdös, P., and Lengyel, B. A. On fundamental functions of Lagrangean interpolation, 828.

Feld, J. M. On certain groups of birational contact transformations, 529.

Feldheim, E. Un problème de la théorie des nombres rattaché aux polynômes de Tschebycheff, 836.

Fialkow, A. The Riemannian curvature of a hypersurface, 253.

Frame, J. S. A symmetric representation of the twenty-seven lines on a cubic surface by lines in a finite geometry, 658.

Frink, A. H., and Frink, O. Polygonal variations, 539.

Frink, O. See Frink, A. H.

Goldstine, H. H. A multiplier rule in abstract spaces, 388.

Green, J. W. A property of harmonic functions in three variables, 548.

Grünwald, G. See Erdös, P.

Hall, M. See Reviews, under Speiser.

Halmos, P. R. Note on almost-universal forms, 141.

Hamilton, H. J. Some theorems on subsequences, 298.

Herzberger, M. See Reviews, under Synge.

Hestenes, M. R. A sufficiency proof for isoperimetric problems in the calculus of variations, 662 .

- See Reviews, under Volterra.

Hildebrandt, T. H. Linear operations on functions of bounded variation, 75.

- See Reviews, under Menchoff, Rothe.

Hollcroft, T. R. Reports of meetings of the American Mathematical Society: October meeting in New York, 3; Annual meeting in Indianapolis, 153; February meeting in New York, 305; A pril meeting in Charlottesville, 463; Summer meeting in New York, 745.

Hyers, D. H. A note on linear topological spaces, 76.

Ingraham, M. H. On certain equations in matrices whose elements belong to a division algebra, 117.

- Report of the November meeting in Iowa City, 7.

Jeffery, R. L. The equivalence of sequence integrals and non-absolutely convergent integrals, 840 .

John, F. A note on the maximum principle for elliptic differential equations, 268.

Jones, F. B. Concerning R. L. Moore's Axiom 5, 689.

Kasner, E. Polygenic functions whose associated element-to-point transformation converts unions into points, 726.

Kasner, E., and DeCicco, J. J. The geometry of the whirl-motion group $G_{6}$ : elementary invariants, 399.

Kraus, C. A. The present status of the theory of electrolytes, 361 .

Lamson, K. W. See Reviews, under Rothe.

Lehmer, D. H. An application of Schläfli's modular equation to a conjecture of Ramanujan, 84 .

Lengyel, B. A. See Erdös, P.

Levine, J. Metric spaces with geodesic Ricci curves, I, 145.

Lewis, F. A. Note on the defining relations for the simple group of order 660, 456.

Longley, W. R. See Reviews, under Hamel.

Lowan, A. N. On the operational determination of two dimensional Green's functions in the theory of heat conduction, 125.

MacLane, S. See Reviews, under Carnap.

MacNeille, H. M. The application of lattice theory to integration, 825 . 
Macphail, M. S. Some iterated integrals in the fractional calculus, 707.

Maddaus, I. On completely continuous linear transformations, 279.

Maker, P. T. The relation of perfect sets of measure zero to certain classes of functions, 846.

Margenau, H. See Reviews, under Cramer.

Menger, K. Non-euclidean geometry of joining and intersecting, 821.

Merriman, G. M. Concerning sets of polynomials orthogonal simultaneously on several circles, 57.

Mersman, W. A. A new summation method for divergent series, 667.

von Mises, R. Note on deduced probability distributions, 81 .

Moore, C. N. See Reviews, under Mandelbrojt, Ser.

Moritz, R. E. On the extended form of Cauchy's condensation test for the convergence of infinite series, 441.

Murnaghan, F. D. See Reviews, under Gamow.

Myers, S. B. See Reviews, under Blaschke, Santalo.

Nakayama, T. A remark on representations of groups, 233.

- A note on the elementary divisor theory in non-commutative domains, 719.

Northrop, F. S. C. See Reviews, under Späth.

Olds, E. G. A moment-generating function which is useful in solving certain matching problems, 407.

Ore, O. On the application of structure theory to groups, 801.

See Reviews, under Hasse, Koksma, Van der Waerden.

Page, L. See Reviews, under Larmor, Pidduck.

Patterson, B. C. See Reviews, under Doehlemann.

Pekeris, C. L. See Reviews, under Ertel.

Pettis, B. J. A note on regular Banach spaces, 420.

Phillips, H. B. Harry Walter Tyler-in memoriam, 596.

Pitcher, E., and Sewell, W. E. Existence theorems for solutions of differential equations of non-integral order, 100.

- A correction, 888.

Poritsky, H. On the boundary condition $\partial u / \partial n+a u=0$ for harmonic functions, 443.

On a mixed boundary condition for harmonic functions, 723.

Putnam, T. M. Reports of meetings of the American Mathematical Society: November meeting in Pasadena, 9; A pril meeting in Berkeley, 461.

Quine, W. V. See Reviews, under Tarski.

Randels, W. C. On the order of the partial sums of a Fourier series, 286. On the absolute summability of Fourier series, 733.

Rasmusen, R. B. Metric properties of the cylinder of Kubota, 674.

Raynor, G. E. See Reviews, under Tricomi.

Rees, P. K. The transforms of Fuchsian groups, 580.

Reid, W. T. A theorem on quadratic forms, 437.

Risselman, W. C. Approximation to a given function by means of polynomials in another given function, 241.

Roberts, J. H. See Reviews, under Malengreau.

Robertson, H. P. See Reviews, under Schaefer.

Robertson, M. S. Multivalent functions of order $p, 282$.

Robinson, R. M. Note on convex regions on the sphere, 115.

Rosenbaum, B. Divisibility of generalized factorials, 566.

Rosser, B. See Reviews, under Hilbert.

Rosser, B., and Walker, R. J. On the transformation group for diabolic magic squares of order four, 416. 
Schaeffer, A. C. See Duffin, R. J.

Schaeffer, A. C., and Duffin, R. J. On some inequalities of S. Bernstein and W. Markoff for derivatives of polynomials, 289.

Schoenberg, I. J. On the Peano curve of Lebesgue, 519.

Sewell, W. E. See Pitcher, E.; Walsh, J. L.

Sheffer, I. M. See Reviews, under Denjoy, Lavrentieff.

Shohat, J. A. See Reviews, under Remes.

Sinkov, A. On generating the simple group $L F\left(2,2^{N}\right)$ by two operators of periods two and three, 449.

Smith, F. C. Relations among the fundamental solutions of the generalized hypergeometric equation when $p=q+1$. I. Non-logarithmic cases, 429 .

Smith, P. A. The topology of transformation groups, 497.

Smithies, F. Completely continuous transformations in Hilbert space, 835.

Snyder, V. See Reviews, under O'Hara.

Sobczyk, A. See Bohnenblust, H. F.

Sokolnikoff, E. S. See Sokolnikoff, I. S.

Sokolnikoff, I. S., and Sokolnikoff E. S. Torsion of regions bounded by circular arcs, 384.

Southall, J. P. C. See Reviews under Carathéodory.

Stone, M. H. The representation of Boolean algebras, 807.

Szász, O. On Fourier series with restricted coefficients, 850.

Szegö, G. See Reviews, under Goursat.

Tamarkin, J. D. See Reviews, under Kaczmarz, Kestelman, Saks, Titchmarsh.

Tang, T. Algebraic postulates and a geometric interpretation for the Lewis calculus of strict implication, 737.

Taub, A. H. Spin representation of inversions, 860.

Taylor, A. E. The resolvent of a closed transformation, 70.

Thrall, R. M. A note on numbers of the form $a^{2}+\alpha b^{2}+\beta c^{2}+\alpha \beta d^{2}, 404$. A polarity of trilinear forms and pencils of bilinear forms, 678.

Thurston, H. S. Matric conjugates in a ring $R(A), 258$.

Trjitzinsky, W. J. Singular point problems in the theory of linear differential equations, 209.

Tukey, J. W. See Boas, R. P.

Vanderslice, J. L. See Reviews, under Cartan.

Vaughan, H. E. On the class of metrics defining a metrisable space, 557

Walker, R. J. See Rosser, B.; see Reviews, under Sauer.

Wall, H. S. On continued fractions representing constants, 94 .

- On the nth derivative of $f(x), 395$.

Walsh, J. L. Note on the curvature of orthogonal trajectories of level curves of Green's functions, 520 .

Walsh, J. L., and Sewell, W. E. Note on degree of trigonometric and polynomial approximation to an analytic function, 865.

Webster, M. S. Orthogonal polynomials with orthogonal derivatives, 880.

Weisner, L. See Reviews, under Carmichael.

Weyl, H. See Reviews, under Cartan, Courant.

White, H. S. Formal synthesis of two periodic correspondences, of period five and seven, respectively, 562.

Whitney, H. See Reviews, under Stöilow.

Whyburn, G. T. A theorem on interior transformations, 414.

Widder, D. V., and Wiener, N. Remarks on the classical inversion formula for the Laplace integral, 573. 
Wiener, N. See Widder, D. V.

Williams, A. R. Birational transformations in 4-space and 5-space, 272.

Wolf, M. C. Transformation of bases for relative linear sets, 716.

Wong, B. C. Enumerative properties of plane connected n-lines, 693.

\section{REVIEWS}

Ackermann, W. See Hilbert, D.

Albert, A. A. Modern Higher Algebra. (The University of Chicago Science Series.) H. W. Brinkmann, 471.

Bachelier, L. Les Lois des Grands Nombres du Calcul des Probabilités. J. L. Doob, 20. Blaschke, W. Integralgeometrie. (Actualités Scientifiques et Industrielles, no. 252.) S. B. Myers, 614 .

Über eine geometrische Frage von Euclid bis Heute. (Hamburger mathematische Einzelschriften, no. 23.) S. B. Myers, 614.

- Vorlesungen über Integralgeometrie. Vol. 1. 2d edition. (Hamburger mathematische Einzelschriften, no. 20.) S. B. Myers, 614.

- Vorlesungen über Integralgeometrie. Vol. 2. (Hamburger mathematische Einzelschriften, no. 22.) S. B. Myers, 614.

Böhm, F. Versicherungsmathematik. Vol. 1. 2d edition. (Sammlung Göschen, no. 180.) E. L. Dodd, 610.

British Association for the Advancement of Science. Mathematical Tables. Vol. 6. Bessel Functions. Part 1. Functions of Orders Zero and Unity. G. R. Clements, 766.

Carathéodory, C. Geometrische Optik. (Ergebnisse der Mathematik und ihrer Grenzgebiete, vol. 4, no. 5.) J. P. C. Southall, 309.

Carmichael, R. D. Introduction to the Theory of Groups of Finite Order. L. Weisner, 178.

Carnap, R. The Logical Syntax of Language. Translated from the German by A. Smeaton (Countess von Zeppelin). (International Library of Psychology, Philosophy, and Scientific Method.) S. MacLane, 171.

Cartan, E. Leçons sur la Théorie des Espaces à Connexion Projective. (Cahiers Scientifiques, vol. 17.) J. L. Vanderslice, 11.

- La Thêorie des Groupes Finis et Continus et la Géométrie Différentielle Traitées par la Méthode du Repère Mobile. (Cahiers Scientifiques, vol. 18.) H. Weyl, 598.

Contributions to the Calculus of Variations, 1933-1937. (Theses submitted to the Department of Mathematics at the University of Chicago.) A. Dresden, 604.

Courant, R., and Hilbert, D. Methoden der mathematischen Physik. Vol. 2. (Grundlehren der mathematischen Wissenschaften in Einzeldarstellungen, vol. 48.) H. Weyl, 602.

Cramer, H. Random Variables and Probability Distributions. (Cambridge Tracts in Mathematics and Mathematical Physics, no. 36.) H. Margenau, 611.

Denjoy, A. Introduction à la Théorie des Fonctions de Variables Réelles. (Actualités Scientifiques et Industrielles, nos. 451-452.) I. M. Sheffer, 21.

Doehlemann, K. Projektive Geometrie. Edited by H. Timerding. (Sammlung Göschen, no. 72.) B. C. Patterson, 318.

Doetsch, G. Theorie und Anwendung der Laplace-Transformation. (Grundlehren der mathematischen Wissenschaften in Einzeldarstellungen, vol. 47.) R. V. Churchill, 476.

Ertel, H. Methoden und Probleme der dynamischen Meteorologie. (Ergebnisse der Mathematik und ihrer Grenzgebiete, vol. 5, no. 3.) C. L. Pekeris, 765.

Gamow, G. Structure of Atomic Nuclei and Nuclear Transformations. F. D. Murnaghan, 611. 
Goursat, E. Propriêtês Générales de l'Équation d'Euler et de Gauss. (Actualités Scientifiques et Industrielles, no. 333.) G. Szegö, 16.

Guillaume, E. See Guillaume, G.

Guillaume, G., and Guillaume, E. Économique Rationnelle. (Actualités Scientifiques et Industrielles, nos. 504-508.) H. T. Davis, 761.

Hamel, G. Integralgleichungen. W. R. Longley, 315.

Hasse, H. Höhere Algebra. II. Gleichungen höheren Grades. 2d edition. (Sammlung Göschen, no. 932.) O. Ore, 178.

Heisenberg, W. See Späth, E.

Hilbert, D. See Courant, R.

Hilbert, D., and Ackermann, W. Grundzige der theoretischen Logik. 2d edition. B. Rosser, 474 .

Hostinsky, B. See Volterra, V.

Huntington, E. V. Postulates for assertion, conjunction, negation, and equality. (Proceedings of the American Academy of Arts and Sciences, vol. 72 (1937), pp. 144.) A. Church, 177.

Kaczmarz, S., and Steinhaus, H. Theorie der Orthogonalreihen. (Monografje Matematyczne, vol. 6.) J. D. Tamarkin, 20.

Kestelman, H. Modern Theories of Integration. J. D. Tamarkin, 616.

Koksma, J. F. Diophantische Approximationen. (Ergebnisse der Mathematik und ihrer Grenzgebiete, vol. 4, no. 4.) O. Ore, 22.

Larmor, J. Origins of Clerk Maxwell's Electric Ideas as Described in Familiar Letters to William Thomson. L. Page, 320.

Lavrentieff, M. Sur les Fonctions d'une Variable Complexe Représentables par des Séries de Polynômes. (Actualités Scientifiques et Industrielles, no. 441.) I. M. Sheffer, 22.

Lévy, P. Théorie de l'Addition des Variables Aléatoires. (Monographies des Probabilités, no. 1.) J. L. Doob, 19.

Malengreau, J. Essai sur les Fondements de la Géométrie Euclidienne. J. H. Roberts, 767.

Mandelbrojt, S. Séries Lacunaires. (Actualités Scientifiques et Industrielles, no. 305.) C. N. Moore, 313.

Mark, H. See Späth, E.

Mattioli, G. D. Teoria Dinamica dei Regimi Fluidi Turbolenti. H. Bateman, 316.

Menchoff, D. Les Conditions de Monogénéitê. (Actualités Scientifiques et Industrielles, no. 329.) T. H. Hildebrandt, 18.

Menger, K. See Späth, E.

Mineur, H. Technique de la Méthode des Moindres Carrés. (Monographies des Probabilités, no. 2.) A. H. Copeland, 766.

Nicolesco, M. Les Fonctions Polyharmoniques. (Actualités Scientifiques et Industrielles, no. 331.) M. A. Basoco, 18.

O'Hara, C. W., and Ward, D. R. An Introduction to Projective Geometry. V. Snyder, 178.

Osgood, W. F. Functions of a Complex Variable. A. A. Bennett, 16.

- Functions of Real Variables. A. A. Bennett, 16.

Pérès, J. See Volterra, V.

Pidduck, F. B. Lectures on the Mathematical Theory of Electricity. L. Page, 612.

Remes, E. On Methods for Obtaining the Best Approximation of Functions, in the Sense of Tchebycheff. In the Ukrainian language. J. A. Shohat, 14.

Roth, E. Axiomatische Untersuchungen zur projektiven, affinen und metrischen Geome- 
trie. (Forschungen zur Logik und zur Grundlagen der exacten Wissenschaften, new series, no. 2.) A. A. Bennett, 479.

Rothe R. Höhere Mathematik für Mathematiker, Physiker, und Ingenieure. IV. Übungsaufgaben mit Lösungen. Formelsammlung. No. 4. Unendliche Reihen. Vektorrechnung nebst Anwendungen. No. 5. Raumkurven und Flachen, Linienintegrale und mehrfache Integrale. No. 6. Gewöhnliche und partielle Differentialgleichungen nebst Anwendungen. T. H. Hildebrandt, 177.

- Differentialgeometrie. Vol. 1. Raumkurven und Anfänge der Flächentheorie. (Sammlung Göschen, no. 1113.) K. W. Lamson, 612.

Russell, B. Principles of Mathematics. 2d edition. F. Creedy, 613.

Saks, S. Theory of the Integral. 2d edition. English translation by L. C. Young. (Monografje Matematyczne, vol. 7.) J. D. Tamarkin, 615.

Santalo, L. A. Integralgeometrie. (Actualités Scientifiques et Industrielles, no. 357.) S. B. Myers, 614 .

Sauer, R. Projektive Liniengeometrie. (Göschens Lehrbücherei, vol. 23.) R. J. Walker, 612.

Schaefer, C. Quantentheorie. (Einführung in die theoretische Physik, vol. 3, no. 2.) H. P. Robertson, 319.

Ser, J. La Réduction des Séries Alternées Divergentes et ses Applications. C. N. Moore, 313.

Smeaton, A. See Carnap, R.

Späth, E., Thirring, H., Mark, H., Heisenberg, W., and Menger, K. Neuere Fortschritte in den exacten Wissenschaften. F. S. C. Northrop, 23.

Speiser, A. Theorie der Gruppen von Endlicher Ordnung. 3d edition. M. Hall, 313.

Steinhaus, H. See Kaczmarz, S.

Stöilow, S. Leçons sur les Principes Topologiques de la Théorie des Fonctions Analytiques. H. Whitney, 758.

Stumpff, K. Grundlagen und Methoden der Periodenforschung. H. Emmons, 762.

Synge, J. L. Geometrical Optics. An Introduction to Hamilton's Method. M. Herzberger, 321.

Tarski, A. Einführung in die mathematische Logik und in die Methodologie der Mathematik. W. V. Quine, 317.

Thirring, H. See Späth, E.

Thomas, J. M. Differential Systems. (American Mathematical Society Colloquium Publications, vol. 21.) S. Bochner, 314.

Timerding, H. See Doehlemann, K.

Tinbergen, J. An Econometric Approach to Business Cycle Problems. (Actualités Scientifiques et Industrielles, no. 525.) H. T. Davis, 761.

Titchmarsh, E. C. Introduction to the Theory of Fourier Integrals. J. D. Tamarkin, 764.

Tricomi, F. Funzioni Analitiche. G. E. Raynor, 610.

- Funzioni Ellittiche. G. E. Raynor, 610.

Van der Waerden, B. L. Moderne Algebra. Vol. 1. 2d edition. O. Ore, 320.

Volterra, V., and Hostinsky, B. Operations Infinitésimales Linêaires. G. Birkhoff, 759.

Volterra, V., and Pérès, J. Théorie Générale des Fonctionnelles. Vol. 1. M. R. Hestenes, 311.

Ward, D. R. See O'Hara, C. W.

Whittaker, E. T. A Treatise on the Analytical Dynamics of Particles and Rigid Bodies with an Introduction to the Problem of Three Bodies. 4th edition. H. E. Buchanan, 316.

Woodger, J. H. The Axiomatic Method in Biology. E. S. Allen, 763. 
Young, L. C. See Saks, S.

Zassenhaus, H. Lehrbuch der Gruppentheorie. Vol. 1. (Hamburger mathematische Einzelschriften, no. 21.) E. T. Bell, 478.

Abstracts of papers presented to the Society, 28, 185, 328, 484, 623, 772.

New Publications, 54, 205, 357, 494, 645, 791.

Notes, $24,180,322,480,617,768$.

\section{NOTES AND OTHER ITEMS}

Academies, Associations, Congresses, and Societies:

American Mathematical Society: Annual Meeting (1937), 153; Auditor's Report, 159; Bôcher Prize, 160, 466; Budget, 4, 159; Bulletin, 1, 2, 749, 768; ByLaws, 4, 749; Colloquium Lectures (1939), 4; Colloquium Publications, 4; Committee on Bôcher Prize, 466; on Colloquium, 467; on Durham Meeting, 466, 749; on Gibbs Lectures, 467, 748; on International Congress (1940), 160, 464, 593, 749; on Meeting Fees, 4, 160; on Nominations, 4; on Publicity, 160, 749; to Select Hour Speakers, 749; on Summer Meeting (1939), 749; Dates and Places of Meetings, 161, 466, 748; Election of Officers, 156; Gibbs Lecture, 154; Invitations for Meetings, 161, 748; Librarian's Report, 159; New Members Admitted, 4, 156, 464, 748; Representatives on the Council of the American Association for the Advancement of Science, 160; at Conference on Problems in Publication of Research, American Philosophical Society, 466; at the Academic Ceremonies, Emory and Henry College, 748; at the Dedication of The Franklin Institute of the State of Pennsylvania, 466; at the Celebration of the One Hundredth Anniversary of Greensboro College, 466; at Conferences on Higher Education and Dedication of Buildings at Louisiana State University, 466; at Semicentennial Celebration of the Founding of Utah State Agricultural College, 748; on the National Research Council, 160; Statistics, 159; Summer Meeting (1938), 745; Transactions, 24, 749; Treasurer's Report, 159.

Associations for the Advancement of Science: American, 4, 153, 154, 155, 160, 181, 768; British, 481, 769.

Academy of Science of Turin, 181; American Academy of Arts and Sciences, 481; American Association of University Professors, 181; American Library Association, 769; American Philosophical Society, 480, 768; Association for Symbolic Logic, 153, 155, 768; Henri Becquerel Foundation, 181; Congresses for the Unity of Science, 768; Cowles Commission for Research in Economics, 322; Econometric Society, 155; Edinburgh Mathematical Society, 24; Franklin Institute, 466; Hirn Foundation, 181; Institute of Mathematical Statistics, 153, 155; International Astronomical Union, 617; International Congress for Applied Mechanics, 480; International Congress for the Unity of Science, 768; International Congress of Mathematicians, 160, 593; International Federation for Documentation, 322; International Institute for the Unity of Science, 768; International Institute of Intellectual Coöperation of the League of Nations, 617; London Mathematical Society, 181, 617; Loutreuil Foundation, 181; Mathematical Association of America, 153, 155, 156, 480; National Research Council, 160; North Carolina Academy of Science, 481; Paris Academy of Sciences, 181; Philosophy of Science Association, 768; Radio Club of America, 24; Rockefeller Foundation, 160; Royal Society of Edinburgh, 24, 769; Society for the Promotion of Engineering Education, 769; Society of Science of Warsaw, 481; Villemot Foundation, 181 
Books, Announcement of New, 467, 480, 768.

Doctorates, American, 322.

Journals:

American Journal of Mathematics, 159, 161; Bulletin of the American Mathematical Society, 1, 2, 749, 768; Mathematische Annalen, 769; Portugaliae Mathematica, 480; Transactions of the American Mathematical Society, 24, 749.

National Research Fellowships, 482, 618.

Papers and Communications Presented to the Society, Authors:*

Adams, C. R., 750 (484); Adkisson, V. W., (786); Agnew, R. P., 5 (43; 765), 307 (185), 469 (328), 755 (639); Akeley, E. S., 459 (354); Alaoglu, L., 162 (29), 459 (196); Albert, A. A., 9 (43; 771), 163 (29), 307 (185), 460 (196), 460 (328); Albert, G. E., 753 (484), (639); Allendoerfer, C. B., 458 (328); Archibald, R. G., 753 (623); Arnold, H. A., 10 (43; 775), 10 (43; 777), 462 (328), 462 (328), (775); Aroian, L. A., 751 (623); Ayres, W. L., 467 (329).

Baer, R., 753 (490), (779), (779), (780); Baker, G. A., 461 (329); Ballou, D. H., 752 (623); Bardell, R. H., 459 (329); Bartels, R. C. F., 458 (329); Basoco, M. A., (780); Baten, W. D., 751 (623); Beckenbach, E. F., 162 (43; 770), 308 (185), 750 (490); Bell, C., (775); Bell, E. T., 10 (43; 774), 10 (43; 776), 10 (43; 777), 164 (29), 462 (330); Bell, P. O., 8 (43; 772), 751 (624); Bergmann, S., 752 (779); Bernstein, B. A., 462 (330); Birkhoff, G., 308 (185), 308 (186), 469 (330), 753 (354); Blake, A., 751 (491); Bliss, G. A., 162 (29); Blumberg, H., 753 (639); Blumenthal, L. M., 8 (43; 771); Boas, R. P., 5 (43; 765), 306 (186), 467 (330); Bochner, S., 306 (186); Brauer, R., 5 (43; 766), 468 (330), 752 (624); Brenner, J. L., 460 (331); Brinkmann, H. W., (772); Bristow, L., (785); Brown, O. E., (780); Browne, E. T., 469 (331); Burington, R. S., (780).

Calkin, J. W., 164 (30), 306 (186); Cameron, R. H., 5 (43; 766); Camp, B. H., 164 (30); Campaigne, H. H., 459 (331); Carpenter, A. F., 468 (331); Carroll-Rusk, E., 468 (350); Carslaw, H. S., 753 (493); Cassity, C. R., 458 (196); Cell, J. W., 469 (331); Chatland, H., 460 (197); Churchill, R. V., 9 (43; 771), 164 (30), 752 (624); Clark, C. E., 753 (625); Clarkson, J. A., 750 (484); Clarkson, J. M., 165 (31); Claytor, W. S., 460 (332); Copeland, A. H., 162 (31); Coral, M., (781); Courant, R., 307 (187), (772), (772); Coxeter, H. S. M., 468 (187), 751 (625); Craig, A. T., 8.(28); Craig, H. V., (781); Curry, H. B., 163 (31); Curtiss, J. H., 5 ( $43 ; 766)$.

Daoust, J. H., 8 (43; 772); Darkow, M. D., (786); Daus, P. H., 10 (43; 776), 462 (332); Davis, H. T., 164 (31); Day, M. M., 752 (625); DeCicco, J. J., 5 (43; 766), 165 (32), 165 (35), 165 (35), 307 (187), 308 (190), 751 (485), 753 (355), 753 (485), (640), (781); Diamond, A. H., 10 (43; 775); Dilworth, R. P., 9 (43; 773), 461 (353), 462 (332), 754 (625), (775); Dines, L. L., 752 (633); Dix, C. H., 462 (332), 462 (333); Dobbie, J. M., 459 (197), (782); Dodd, E. L., 5 (43; 627); Douglas, J., 754 (487), 754 (487), 754 (491), 754 (491), (640), (640), (640), (785), (787); Dresden, A., 307 (187); Dresher, M., 306 (188); Dribin, D. M., 468 (333); Duffin, R. J., 459 (333), 459 (349), 752 (626); Dunford, N., 308 (197); Duren, W. L., 165 (32); Dushnik, B., 458 (333); Duthie, W. D., (640).

Earl, J. M., 459 (334); Eaton, J. E., 306 (188); Eisenhart, C., 164 (32); Emch, A., 458 (197), 751 (626); Ewing, G. M., 8 (43; 772).

* Numbers within parentheses refer to abstracts, and those outside parentheses refer to titles as listed in reports of meetings. 
Feld, J. M., 307 (50), 469 (334); Fialkow, A., 5 (43; 628), 307 (50), 754 (491); Ficken, F. A., (772); Flexner, W. W., 162 (32), 753 (626), 754 (627); Flood, M. M., 163 (33); Ford, L. R., 458 (336); Forsyth, C. H., 165 (43; 770); Fort, T., 307 (188), (773); Foster, A. L., 461 (334); Frame, J. S., 307 (188); Frankel, L. R., 165 (34); Franklin, P., 5 (43; 767), 467 (334); Friedman, B., 459 (335), 753 (627); Friedrichs, K. O., 750 (627); Frink, A. H., 467 (198); Frink, O., 467 (198).

Gage, W. H., 754 (493); Garabedian, H. L., 460 (335); Gentry, F. C., 754 (485), 754 (485); Gerhardt, H., 751 (493); Givens, J. W., 306 (189), (782); Glenn, O. E., 753 (627); Goldstine, H. H., 9 (43; 771), 459 (335); Gorn, S., 750 (335); Green, J. W., 9 (43; 773), 308 (51), 462 (336); Green, L., 754 (628); Griffin, F. L., 307 (189); Griffiths, L. W., 165 (33).

Haberzetle, M. B., 459 (336); Hahn, J. W., 458 (336); Hall, D. W., 9 (43; 774), 461 (336), (773), (776); Hall, M., 469 (337), (628); Hall, N. A., 9 (43; 773), 461 (337), 752 (488); Halmos, P. R., 164 (43; 770), 468 (337); Halperin, I., 307 (189); Hamilton, H. J., 9 (43; 773), 469 (337), 469 (338), (776); Hamilton, O. H., 469 (338), 753 (492); Harkin, D. C., 163 (33), 751 (628); Harrold, O. G., (776), (776); Hartman, P., 5 (43; 777), 308 (189); Hedlund, G. A., 467 (345); Heins, M. H., (787); Helmer, O., 163 (33), 459 (338); Henderson, A., 754 (492); Henle, P., 163 (34); Herzberger, M., 307 (189); Hestenes, M. R., 460 (338); Hille, E., 308 (198), 308 (198), 468 (339); Hoel, P. G., 462 (339); Hollcroft, T. R., 163 (34), 468 (339), 751 (628), (773); Hopkins, C., 469 (198), 751 (628); Hotelling, H., 165 (34), 751 (629); Hull, R., 460 (339); Huntington, E. V., 164 (51), 751 (492); Hyers, D. H., 750 (629), (777).

Ingraham, M. H., 460 (340); Ingram, W. H., 6 (43; 468), 307 (43; 780).

Jackson, D., 8 (43; 772$)$, 750 (629); Jackson, S. B., 458 (340); Jacobson, N., 468 (340), 469 (340); Jaeger, J. C., 753 (493); James, G., 9 (43; 774), 462 (341); James, R. D., 461 (341), (776); John, F., 459 (341); Jones, B. W., 469 (341); Jones, F. B., 460 (199), (787), (788); Jones, H. E., 164 (31).

Kagno, I. N., 469 (341); Kales, M. L., 459 (342); Kamke, E., 752 (629); van Kampen, E. R., 469 (342); Kasner, E., 165 (35), 165 (35), 308 (190), 753 (355), 754 (355), (640); Kawaguchi, A., 165 (43; 769); Kazarinoff, D. K., (782); Kelley, J. L., 467 (190), (788); Kenney, J. F., (782); Kershner, R. B., 8 (43; 773), 308 (189), 458 (342); Knebelman, M. S., 468 (342); Koehler, F., 8 (43; 772); Kolchin, E. R., 754 (488); Krall, H. L., 468 (199).

Lamoreau, F. L., (641); Lamson, K. W., (641); Lancaster, O. E., 164 (43; 778), 165 (35), 469 (199); Lanczos, C., (783); Langer, R. E., 460 (356); Langford, C. H., 163 (36); Lasley, J. W., 754 (492); Latshaw, V. V., 6 (43; 767); Lehmer, D. H., 306 (190), 754 (630); Leighton, W., 750 (488), 750 (630), (788); Lengyel, B. A., 750 (489); Lester, C. A., 5 (43; 628); Levi, H., 306 (191), 753 (630); Levinson, N., (788), (789); Levitzki, J., 165 (36); Lewy, H., 462 (342); Lorch, E. R., 306 (51), 752 (486); Lowan, A. N., 6 (43; 767), 308 (191), (641), (641), (774), (774); Lubben, R. G., 162 (36), 753 (630).

McCoy, N. H., 165 (37), 306 (191), 754 (492); McDaniel, R. R., 469 (343); McEwen, W. H., 165 (37), 754 (631); McShane, E. J., 162 (52), 468 (343); MacColl, L. A., (642); MacDonald, J. K. L., 750 (631), 754 (631); MacKay, R., 753 (631); MacLane, S., 164 (37), 460 (199), 460 (343), 754 (632), (783); MacNeille, H. M., 162 (38); Maker, P. T., 468 (200); Mancill, J. D., 460 (200), (774); Manning, D., (642); Martin, W. T., 307 (191); Menger, K., 163 (38), 165 (39), (785); Merriman, G. M., 754 (632); Michal, A. D., 10 (43; 775), 10 
(43; 777), 462 (343), 462 (344), 462 (344), (777), (777); Milgram, A. N., 162 (38), 165 (39); Miller, E. W., 460 (344); Milne, W. E., 162 (39); Montgomery, D., 5 (43; 767$)$, 165 (37), 753 (489); Moore, C. N., 467 (344); Moore, M. G., 162 (40), 165 (39), 459 (345); Morrey, C. B., 307 (192), 468 (345); Morse, A. P., 165 (40), 755 (486); Morse, M., 467 (345), 750 (632); Moskovitz, D., 752 (633); Murdoch, D. C., 468 (345); Murnaghan, F. D., 469 (200), 469 (200); Murray, F. J., 308 (52); Musselman, J. R., (783); Myers, S. B., 163 (40).

Nakayama, T., 5 (43; 768), 306 (192), 468 (346), 751 (633); Nesbitt, C. J., 5 (43; 766), 165 (40), 468 (346), (783); Netzorg, D. L., 468 (346); Niven, I., 459 (346).

Oberg, E. N., 755 (633); O'Connor, R. E., 6 (43; 628), 752 (633); Oldenburger, R., 5 (43; 768), 165 (41), 470 (346), 751 (634), (784); Olds, E. G., 164 (43; 779); Ore, O., 306 (188); Oxtoby, J. C., 467 (347).

Palermo, A. J., 750 (634); Pall, G., 752 (633), 755 (634); Palmer, T. P., 751 (634); Paxson, E. W., 162 (41), 753 (492); Peebles, G. H., (785); Perlis, S., 460 (356); Pettis, B. J., 6 (43; 768$), 307$ (192), 755 (486); Pitcher, A. E., (784); Poor, V. C., 164 (43; 779), 165 (43; 779); Popoff, K., 752 (642); Poritsky, H., 755 (643); Price, G. B., 162 (41), 755 (635); Puckett, W. T., 467 (201), 470 (201).

Rademacher, H. A., 470 (347), 755 (635); Rad6, T., 164 (43; 769); Rainich, G. Y., 163 (42); Rainville, E. D., 459 (347), 460 (347), (784); Randels, W. C., 8 (43; 770), 164 (42), 460 (335); Randolph, J. F., 162 (43; 769), 306 (192); Reade, M., 308 (185), 750 (490); Reed, F. W., 467 (348); Reid, W. T., 165 (42), 752 (635); Riabouchinsky, D., 751 (636); Rietz, H. L., 8 (28); Rigby, F. D., 8 (28); Rinehart, R. F., 164 (42); Risselman, W. C., 166 (43); Ritt, J. F., 470 (348); Robbins, H. E., 753 (636); Roberts, J. H., 467 (348), 755 (643), 755 (643); Robertson, M. S., 470 (201), (786); Robinson, L. B., 6 (43; 768), 308 (193), 470 (348); Robinson, R. M., 10 (43; 776), (644); Rosser, J. B., 163 (43), 166 (43), 306 (193); Rothe, E. H., 8 (43; 777), 162 (43), 458 (349), (785).

Safford, F. H., 6 (43; 476); Sagen, O. K., 306 (193); Schaeffer, A. C., 459 (349); 752 (626); Schilling, O. F. G., 162 (43), 166 (44), 166 (44), 470 (349), (789); Schoenberg, I. J., 163 (44), 468 (349), 470 (350); Schwartz, H. M., 470 (350); Schweigert, G. E., 9 (43; 774), 753 (636); Schweitzer, A. R., 166 (43; 769), 755 (489), (789); Scott, W. T., 750 (630); Sealander, C. E., 8 (28); Seidel, W., 308 (193), 470 (350); Serghiesco, S., 752 (636); Sewell, W. E., 164 (45), 166 (43; 779), 470 (202), 470 (202), 752 (493); Shaw, A. A., 9 (43; 774), (777), (777); Shiffman, M., 752 (637), 755 (637); Shohat, J. A., 470 (350), (774); Simmons, H. A., 166 (45); Smiley, M. F., 162 (29), 162 (45); Smith, C. D., 751 (637); Smith, F. C., 164 (43; 779); Snyder, V., 468 (350); Sokolnikoff, E. S., 166 (45), 458 (351); Sokolnikoff, I. S., 166 (45), 458 (351); Staniland, A. E., 5 (43; 625); Starkey, D. M., 164 (46); Steenrod, N. E., 163 (40), 467 (348); Stephens, E., 166 (46); Stoker, J. J., 307 (194), 308 (194); Strodt, W. C., 307 (194); Struik, R. R., 5 (43; 778), 6 (43; 778); Suckau, J. W. T., 458 (351); Swinford, L. H., 462 (351); Szász, O., 163 (46), 308 (194), 460 (351).

Taub, A. H., 752 (490), 755 (490); Taussky, O., 166 (43; 780), 755 (487); Taylor, A. E., 307 (195), 308 (194), (778), (778), (778); Thrall, R. M., 459 (202); Thurston, H. S., 163 (47), 755 (638), 755 (638); Tintner, G., 8 (29); Tompkins, C. B., 163 (47); Tornheim, L., 459 (352), 460 (352); Torrance, C. C., 162 (52); Torrance, E. M., 752 (638); Trjitzinsky, W. J., 162 (47), 752 (638), (790); Tucker, A. W., 162 (47), 467 (352); Turrittin, H. L., 461 (352).

Ulam, S. M., 307 (195), 308 (195), 467 (347), (786); Uspensky, J. V., 461 (352).

Valentine, F. A., (778); Vaughan, H. E., 162 (48). 
Wagner, R. W., 459 (353); Walker, R. J., 166 (43), 306 (193); Wall, H. S., 163 (48); Wallace, A. D., 467 (202), 470 (202), 470 (203), (773); Walsh, J. L., 308 (193), 752 (493); Ward, M., 10 (43; 776), 308 (195), 461 (353); Warschawski, S. E., 164 (48); Webb, D. L., 163 (49); Weisner, L., 755 (643), (643), (775); Welch, F. P., 459 (203); Weyl, F. J., 468 (353); Weyl, H., 468 (353); Wheeler, A. H., 468 (353), 751 (638); Whitney, H., 307 (196); Whyburn, G. T., 163 (49), 166 (49), 470 (203); Whyburn, W. M., 162 (49); Widder, D. V., 5 (43; 765); Wiener, N., 307 (191); Wilcox, L. R., 162 (45), 163 (50); Wilder, R. L., 460 (332); Williams, W. L., 755 (490); Williamson, J., 469 (203); Wintner, A., 469 (342); Wishard, A., 164 (53); Wolf, L. A., 752 (639); Wolf, M. C., 163 (50), 752 (639); Wyman, M., (779).

Zariski, O., 469 (354); Zippin, L., 5 (43; 767), 753 (489); Zorn, M., 10 (43; 775), 462 (354); Zuckerman, H. S., 308 (204), 468 (347), 470 (347).

\section{Personal Notes:}

Adams, C. R., 160; Ader, O. B., 322; Agnew, R. P., 481, 768; Ahlfors, L. V., 618; Albert, A. A., 4, 24, 155, 180, 181, 458, 768; Albert, G. E., 621; Alexander, H. W., 26; Allen, R. B., 25, 327; Allen, R. G. D., 322; Allendoerfer, C. B., 322, 483; Alrich, G. F., 322; Alsberg, C. L., 326; Amaldi, E., 182; Amato, V., 182; Archibald, H. F., 771; Archibald, R. C., 160, 768; Archibald, R. G., 183, 768; Armstrong, E. H., 24; Arnold, H. E., 771; Artin, E., 24, 180, 181, 183, 619; Ayres, H. C., 771; Ayres, W. L., 4, 156, 749.

Baer, R., 25, 180, 464, 619, 768; Bailey, A. H., 26; Baker, F. E., 322; Baker, R. P., 8, 322; Bardell, R. H., 619; Bargmann, V., 771; Barkas, W. H., 771; Bartels, R. C. F., 621; Basoco, M. A., 768; Bateman, H., 768; Beal, J. L., 153; Beckenbach, E. F., 768; Bell, E. T., 9, 160, 617, 768; Bell, P. O., 326; Bennett, A. A., 747; Bennett, T., 482; Benson, S. W., 480; Bergmann, P. G., 771; Bernadini, G., 182; Bernamont, J., 181; Berry, A. C., 768; Berzolari, L., 181; Bethenod, J., 181; Bibb, S. F., 619; Bird, M. T., 482; Birkhoff, G., 24, 160, 180, 463, 482; Birkhoff, G. D., 24, 154, 155, 466, 481, 618, 768, 769; Birkhoff, Mrs. G. D., 160; Bishop, M. L., 323; Blake, A., 323; Blaschke, W., 617; Blichfeldt, H. F., 461; Bliss, G. A., 7, 160, 457, 458, 466; Blumberg, J. O., 184; Boas, R. P., 323, 618; Bohr, N., 771; Bond, J. D., 184; Bonnet-Sainturier, R., 181; Borsuk, K., 481; Boswell, J. M., 771; Bourgin, D. G., 25; Bowden, J., 306; Bower, J. W., 482; Brady, C. P., 622; Brahana, H. R., 24, 768; Brandt, A. E., 183; Brauer, R., 180, 768; Brenke, W. C., 7; Brillouin, L., 181; Brown, A. B., 183; Brown, E. W., 622; Browne, E. T., 463; Burgaud, M., 181; Burkhardt, F., 481; Busemann, H., 771 ; Bush, V., 618; Bushey, J. H., 619; Bussey, W. H., 458; Butler, N. M., 747.

Cairns, S. S., 183; Cairns, W. D., 155; Calkin, J. W., 323; Cameron, R. H., 747; Campaigne, H. H., 771; Campbell, W. W., 622; Campedelli, L., 182; Cannon, B., 481; Carlitz, L., 466; Carmichael, R. D., 466; Carrière, Z., 181; Carroll-Rusk, E., 482; Cartan, E. J., 182; Cartwright, M. L., 181; Cassity, C. R., 771; Cattell, J. M., 155; Cell, J. W., 482; Chanler, J. H., 26; Chatland, H., 323; Chevalley, C., 771; Chittenden, E. W., 7, 24, 768; Church, A., 155; Church, R., 619; Churchill, R. V., 482; v. Ciriacy-Wantrup, S., 322; Cisotti, U., 182; Clark, C. E., 621; Clarkson, J. A., 768; Clarkson, J. M., 619; Clifford, A. H., 771; Coble, A. B., 24, 748, 749, 768; Cohen, A., 768; Cohen, L. W., 770; Coleman, A. J., 480; Coleman, W. B., 771; Conklin, E. G., 154; Cooley, H. R., 323; Copeland, A. H., 7; Copeland, L. P., 160; Cotton, A., 181; Couderc, P., 181; Coulson, C. A., 618; Courant, R., 768; Cowgill, A. P., 621; Cowles, A., 322; 
Craig, A. T., 183; Crawford, R. T., 619; Cummings, L. D., 618; Currier, C. H., 618; Curry, H. B., 24, 619, 768, 771; Curtiss, D. R., 2, 155.

Darmois, G., 181; Dauvillier, A., 181; Davis, H. T., 322; Davisson, S. C., 619; Decaux, B., 181; DeCicco, J. J., 771; DeLury, D. B., 183; Denbow, C. H., 323; Denjoy, A., 769; Derry, D., 26, 621; Diamond, A. H., 25, 619; Dickson, L. E., 180; Dieckmann, C. H., 323; Dobbie, J. M., 483; Dodd, E. L., 24, 322; Doob, J. L., 24, 25, 768; Dorroh, J. L., 770; Douglas, J., 771; Douglass, R. D., 482; Downing, R. H., 771; Dresch, F. W., 323; Dresden, A., 160; Dresher, M., 323, 326; Dribin, D. M., 180, 771; Dunford, N., 747, 768; Duren, W. L., 25, 768; Durfee, W. H., 482; Dwyer, W. A., 323.

Eberhart, P., 26; Eddington, A., 617; Elliott, W. W., 748; Emch, A., 768; Enriques, F., 617; Erdös, P., 771; Erickson, C. G., 483; Erskine, W. H., 619; Evans, G. C., 9, 155, 461, 748, 768; Evans, H. P., 749; Ezekiel, M., 322.

Favre, K., 618; Fermi, E., 182; Ferrari, C., 480; Ferry, F. C., 769; Ficken, F. A., 26; Field, P., 183; Filon, L. N. G., 184; Finan, E. J., 619; Fischer, C. H., 183; Fisher, A., 322; Fisher, F. G., 323; Fiske, T. S., 747; Fite, W. B., 25, 747; Fitting, H., 771; Fitzpatrick, J. D., 483; Flood, M. M., 322; Fobes, M. P., 483; Ford, L. R., 768; Ford, W. B., 768; Fort, T., 2, 748; Foster, A. L., 482; Foster, I. G., 26; Fouché, M., 181; Fowler, R. H., 618; Frankel, S. P., 480; Franklin, C. S., 480; Franklin, P., 156, 160, 183, 464, 768; Fry, T. C., 768; Fuller, G., 26; Fulton, D. G., 26.

Gaffney, J., 25; Galbraith, A. S., 26, 323; Galbraith, E., 26; Garabedian, C. A., 326; Garabedian, H. L., 768; Garner, L. L., 25; Gavett, G. I., 25; Gay, H. J., 619; Gentile, G., 182; Gentry, F. C., 25, 323, 770; Gergen, J. J., 466, 467; Getchell, B. C., 153; Giacobini, M., 181; Gill, B. P., 156, 770; Glover, J. W., 482; Gödel, K., 771; Godfrey, E. L., 184; Goldstein, S., 769; Goldstine, H. H., 768; Gorn, S., 768; Gottlieb, M. J., 323; Goudey, R., 181; Graf, U., 618; Grant, A. M. C., 323; Graustein, W. C., 160, 768; Graustein, Mrs. W. C., 160; Graves, C. H., 483; Graves, L. M., 2, 156, 619; Green, J. W., 482; Green, L., 323; Greenwood, J. A., 768, 770; Griffin, F. L., 322; Griffiths, L. W., 770; Grove, V. G., 768; Gundersen, C., 483.

Haack, W., 24; Hacker, S. G., 25; Haldane, J. B. S., 24, 617; Hale, G. E., 483; Hall, D. W., 482; Hall, N. A., 621; Halmos, P. R., 771; Hamilton, O. H., 323; Hammer, P. C., 621; Harkin, D. C., 619; Harrington, W. J., 26; Hartkemaier, H. P., 322; Hartman, P., 771; Hartung, M. L., 619; Harvey, G. G., 482; Hatcher, T. W., 619; Hausmann, B. A., 323; Haviland, E. K., 620; Hazeltine, A., 24 ; Hedberg, E. A., 770; Hedge, L. B., 483; Hedlund, G. A., 2, 747, 771; Hedrick, E. R., 1, 2, 4, 9, 160; Heins, A. E., 323; Hennel, C. B., 25; Hildebrandt, M., 155; Hildebrandt, T. H., 154, 155, 156, 458, 464, 747; Hille, E., 156, 160; Hlavatý, V., 457; Hodgkinson, J., 181; Hoel, P. G., 26; Hölder, O., 27; Hollcroft, T. R., 466; Holly, M. A., 483; Hoskins, L. M., 27; Householder, A. S., 25, 323; Hoyle, V. A., 25; Hull, R., 180, 620; Huntington, E. V., 24, 155, 180; Hurewicz, W., 771; Hurwitz, W. A., 768; Hutchings, W. L., 620; Hydeman, W. R., 26; Hyers, D. H., 324.

Iglish, R., 24; Ikenberry, J. E., 324; Ingraham, M. H., 4, 156, 160, 180, 181, 458, 749; Irwin, F., 482; Iyer, V. G., 770.

Jackson, D., 7, 160, 457, 768; Jackson, S. B., 324; Jacobson, B. A., 480; Jacobson, N., 180, 768, 770; Jäger, G., 326; James, R. D., 768; Jeffery, G. B., 181 ; Jeffery, R. L., 768; Jennings, W., 622; Joffe, S. A., 4, 159, 160; Johannes, K., 26; Johnson, E., 183; Johnston, F. E., 620; Jones, B. W., 771; Jones, G. M., 620; Jones, H. E., 322; Joseph, M., 322. 
Kaltenborn, H. S., 771; Kaplansky, I., 480, 481; Keeler, I. F., 25; Kennedy, E. C., 324; Kershner, R. B., 324; Kerst, D. W., 324; Kersten, H. J., 482; Ketchum, P. W., 25; Kimball, B. F., 482; Kimball, F. H., 620; Kincaid, W. M., 480; King, L. D. P., 324; Kline, J. R., 156, 181, 466; Kline, M., 621; Knaster, B., 481; Knebelman, M. S., 463, 770; Kneschke, A., 481; Knowler, L. A., 26, 324; Knox, R. H., 326; Kohlmetz, D. B., 324; Koopman, B. O., 24; Krathwohl, W. C., 620; Kraus, C. A., 154; Kunz, J., 622; Kusner, J. H., 482.

Lacombe, M., 622; Lagrula, J., 181; Lainé, P., 181; Lancaster, O. E., 324; Landau, E., 326; Lane, E. P., 768; Lang, G. B., 621; Langer, R. E., 155, 156, 160, 458, 747, 768; La Paz, L., 768; Lasley, J. W., 481; Latimer, C. G., 4, 25, 180, 457; Lawton, W. S., 620; Leavens, D. H., 322; Lefschetz, S., 180, 481; Legvold, S., 25; Lehmer, D. H., 481, 768; Lehmer, D. N., 2, 768, 771; Leighton, W., 768; Lemaitre, G., 24, 183; Leray, J., 181; Lerner, A. P., 322; Lesage, A., 181; Lester, C. A., 324; Lettenmeyer, F., 24 ; Leuschner, A. O., 618; Levi-Civita, T., 182, 617; Levinson, N., 768; Lévy, P., 181; Lewy, H., 768; Lippman, C. W., 480; Littlewood, J. E., 617; Lodge, A., 194; Longacre, W. A., 26; Longley, W. R., 2; Lorch, E. R., 156, 747; Lowenstein, L. L., 324; Luck, J. J., 771; Luc-Verbon, M., 181; Luther, H. A., 324; Lyle, R. R., 26.

McCracken, E. C., 620; McDaniel, R. R., 326; McIntire, F., 322; McShane, E. J., 155, 768; MacDonald, J. K. L., 770; MacDuffee, C. C., 5, 180; MacGregor, C. W., 183; MacLane, S., 24, 160, 180, 620, 749, 768; MacNeille, H. M., 464, 482; Maddaus, I., 26; Maier, W., 322; Maillard, L., 622; Majorana, E., 182; Maker, P. T., 483, 771; Malavard, L., 181; Mandelbrojt, S., 618; Mania, B., 182; Manning, D., 324, 771; Marchaud, A., 181; Maroni, A., 182; Marshall, C. E., 770; Marshall, W., 156; Martin, W. T., 160, 482; Mason, R. G., 26; Maurain, C., 181; May, K., 770; Mayer, J., 322; Mayer, W., 771; Mazurkiewicz, S., 481; Meder, A. E., 4, 159, 160; Mehlenbacher, L. E., 184, 326; Mendelsohn, N. S., 480; Mendershausen, H., 322; Menger, K., 5, 24, 180, 183, 464, 483; Michal, A. D., 156, 461; Miles, H. J., 25; Milgram, A. N., 180, 324; Miller, D. S., 26; Miller, H. C., 184; Milne, E. A., 181; Miranda, C., 182; Mitchell, A. C. G., 620; Mitchell, H. H., 768; Mitchell, W. C., 184, 324; Modesitt, V., 324; Moine, M., 181; Montgomery, D., 620, 747, 768; Moore, C. N., 4, 160, 749, 768; Moore, G. E., 25 ; Moore, R. L., 154, 155, 747, 748; Moreland, E. L., 620; Morley, F., 27, 161, 167; Morris, R., 748; Morse, A. P., 324, 771; Morse, M., 160, 464, 768, 770; Moscovitch, N. A., 324; Mosesson, Z. I., 324; Moulton, E. J., 457; Moulton, F. R., 155, 768; Munro, G. C., 26; Murdoch, D. C., 182, 324, 483; Murnaghan, F. D., 160; Murray, F. J., 483; Myers, S. B., 620.

Nakayama, T., 771; Nesbitt, C. J., 325, 483; Netzorg, D. L., 26; v. Neumann, J., 24, 156, 180, 464, 617; Neyman, J., 322; Nikliborc, W., 481; N $\phi$ rlund, N. E., 617; Norton, H. W., 322; Novak, J. D., 26.

Oberg, E. N., 26; Olshen, A. C., 325; Olson, E. J., 621; Ore, O., 24, 180, 464, 768; Orlicz, W., 481; Ostrofsky, M., 325; Ott, E. R., 183.

Palmer, T. P., 26; Palmquist, K. L., 26, 325; Paxson, E. W., 26, 325; Pence, S. E., 325; Pepper, P. M., 26, 325, 483; Perkins, D. T., 483; Perlin, I. E., 26; Pettis, B. J., 325, 481; Pfeiffer, V. W., 771; Phillips, H. B., 306; Phillips, Mrs. H. B., 160; Picone, M., 182; Pillai, S. S., 769; Pipes, L. A., 483; Pitcher, A. E., 621, 768; Pollard, H. S., 620; Post, E. L., 183; Prenowitz, W., 620; Price, G. B., 160, 747, 749; Pryce, M. H. L., 325; Puckett, W. T., 325, 622; Putnam, J. W., 155, 156; Putnam, T. M., 156.

Rabi, I. I., 771; Racah, G., 183; Radakovič, T., 327; Rademacher, H. A., 768; Rainich, G. Y., 768; Ramamurti, B., 769; Randels, W. C., 620; Randolph, 
J. F., 26, 770; Ranzi, I., 183; Ratti, G. A., 156; Rawhouser, R., 621; Rawlins, C. H., 620; Reed, V. D., 322; Rees, H., 325; Reilly, J. F., 8; Rellich, F., 24; Richards, H. C., 618; Richardson, R. G. D., 156, 160; Richtmyer, F. K., 618; Rietz, H. L., 2, 7; Risselman, W. C., 26; Ritt, J. F., 25, 156, 160, 306, 747, 768; Ritter, E., 325; Ritter, E. K., 620; Roberts, J. H., 463, 466; Robertson, H. P., 621; Robbins, H. E., 771; Rodabaugh, L. D., 184; Rogers, W. O., 184; Roos, C. F., 322; Rosenfeld, L., 771; Rosser, J. B., 24; Roth, W. E., 26; Rothe, E., 621; Roure, H., 181; Royall, N. N., 770; Ruse, H. S., 769; Russell, H. N., 618; Rutherford, E., 27.

Sackett, R. L., 769; Sagen, O. K., 26; Sanders, S. T., 466; Sanger, R. G., 458; Sauer, R., 24; Savornin, A., 181; Schilling, O. F. G., 180; Schlauch, W. S., 483; Schmidt, Harry, 618; Schmidt, Hermann, 619; Schneckenburger, E. R., 771; Schoenberg, I. J., 747; Schotland, J. H., 325; Schultz, H., 322; Schweigert, G. E., 622; Schwid, N., 184; Scorza, G., 181, 182; Secrist, H., 322; Seidel, W., 184, 768; Seifert, H., 25; Senior, J. K., 180; Serbin, H., 325, 771; Sermon, T. C., 771; Service, J. H., 771; Sewell, W. E., 26, 768; Shanks, M. E., 771; Sheffer, H. M., 771; Sheffer, I. M., 768; Sherman, S., 26; Shewhart, W. A., 155, 617; Shiffman, M., 771; Shohat, J. A., 182, 768; Shreve, D. R., 483; Siegel, C., 322; Sierpiński, W., 481; Sigley, D. T., 621; Simond, R. G., 483; Slepian, J., 483; Smiley, C. H., 483; Smiley, M. F., 325, 621; Smith, E. R., 8; Smith, F. C., 621; Smith, G., 621; Smith, P. A., 2, 4, 156, 306; Smith, R. E., 771; Smith, S. R., 26; Snyder, V., 483, 768, 770; Sokolnikoff, E. S., 749; Soleillet, P., 181; Sorgenfrey, R. H., 184; Southard, T. H., 184; Stalsinger, J., 480; Stanley, G. K., 771; Stecker, Mrs. O. H., 184; Stehn, J. R., 325; Stelson, H. E., 26; Stephenson, S. A., 771; Stewart, S. W., 26; Stipe, C. G., 771; Stokes, R. W., 466; Stone, M. H., 4, 24, 160, 180, 464, 481, 748; Stopher, E. C., 325; Stouffer, E. B., 7, 24, 154; Strodt, W. C., 483; Struik, D. J., 3, 768; Sugar, A., 621; Sullivan, M. M., 184; Swain, R. L., 184; Synge, J. L., 156, 768; Szász, O., 24, 768; Szegö, G., 621, 768.

Tamarkin, J. D., 4, 466, 467; Tate, J. T., 618; Taylor, A. E., 483; Taylor, W. C., 326, 621; Terracini, A., 182; Thomas, G. B., 26; Thomas, J. M., 768; Thomas, T. Y., 4, 621, 768; Thomson, E., 480; Thomson, J. J., 322; Thorndike, E. L., 24; Thrall, R. M., 325; Timoshenko, S., 618; Tingey, V. H., 748; Tintner, G., 322; Tippett, L. H. C., 617; Tompkins, C. B., 621; Tonelli, L., 181, 182; Torrance, C. C., 621, 771; Torrance, Mrs. C. C., 771; Traber, R. E., 621; Tricomi, F., 182; Trjitzinsky, W. J., 7, 156, 768; Trumpler, R. J., 621; Tucker, A. W., 306, 483; Tucker, C. B., 483; Tuller, A., 325; Tulloch, L. H., 771; Tyler, H. W., 327.

Uhlenbeck, G. E., 481; Ulam, S. M., 24; Ulbricht, E., 322.

Vahlen, T., 322; Valentine, F. A., 325; Valentine, R. H., 325; Vandiver, H. S., 768; Van Schaack, G. B., 621; Van Vleck, J. H., 160, 769; Vassy, E., 181; Veblen, O., 160; Volterra, V., 617.

Wagner, R. W., 326; Wald, A., 322; Walker, R. J., 483, 768; Wallman, H., 326, 771; Walsh, J. L., 4, 747, 768; Ward, L. E., 8; Warnock, W. G., 771; Warschawski, S. E., 26, 621, 768; Watkins, R. J., 322; Webster, M. S., 483; Wegner, K. W., 771; Wehausen, J. V., 621; Weinberg, D., 181; Weiss, M. J., 621; Welch, F. P., 326; Welker, E. L., 483; Wells, N. W., 621; Welmers, E. T., 326; West, J. M., 184; de Wet, J. S., 481, 771; Weyl, H., 326, 749, 768; Whitehead, J. B., 621; Whitney, H., 305; Whyburn, G. T., 155, 467, 768; Whyburn, W. M., 26, 154, 461, 483; Wick, G. C., 183; Widder, D. V., 160, 768; Wiener, N., 5, 749, 768; Wierenga, H., 26; Wigner, E. P., 483, 617; Wilcox, L. R., 622; 
Wilder, R. L., 24, 768; Wildermuth, R. B., 26; Wilks, S. S., 617; Williams, K. P., 153, 621; Williamson, J., 180, 768; Wilson, J. S., 464; Wilson, R., 769; Winton, L. S., 326, 621; Wishard, A., 622; Wolf, M. C., 184; Wood, F., 461; Worden, O. E., 184; Working, E. J., 322; Wray, W. D., 26; Wrench, J. W., 483; Wylie, S., 326, 619.

Yntema, T. O., 322; York, V. O., 26; Yost, F. L., 326.

Zariski, O., 180; Zippin, L., 621, 768.

Prizes and Medals:

Armstrong Medal and Citation for 1937, 24; Henri Becquerel Foundation Award, 181; Binoux Prize, 181; Bordin Prize, 181; Bressa Prize for 1937, 181; Clément-Félix Prize, 181; Commonwealth Club of California Medal, 617; De Morgan Medal, 617; James Alfred Ewing Medal, 480; Francoeur Prize, 181; François Hebert Prize, 181; Hirn Foundation Award, 181; Hughes Prize, 181; Kastner-Boursault Prize, 181; Keith Prize, 769; Kelvin Medal for 1938, 322; Lalande Prize, 181; Lamme Medal, 769; Loutreuil Foundation Award, 181; Montyon Prize, 181; Petit D'Ormoy Prize, 181; Pierson-Perrin Prize, 181; Gaston Planté Prize, 181; Poncelet Prize, 181; G. de Pontecoulant Prize, 181; Henri de Parville Prize, 181; William Lowell Putnam Mathematical Prize, 480; Ramanujan Memorial Prize of Madras University, 769; Saintour Prize, 181; Villemot Foundation Award, 181; Benjamin Walz Prize, 181; Weldon Memorial Prize, 617.

Universities and Technical Schools:

University of Athens, 24; University of California at Berkeley, 480; University of Chicago, 180; University of Cincinnati, 4; Columbia University, 480; Emory and Henry College, 748; Greensboro College, 466; Harvard University, 481; Institute for Advanced Study, 160, 769; Louisiana State University, 161, 466; Madras University, 769; University of Notre Dame, 24, 180; Ohio State University, 161; University of Toronto, 480; Utah State Agricultural College, 748. 